\title{
Time-Dependent Reversal of Long-Term Potentiation in Area CA1 of the Freely Moving Rat Induced by Theta Pulse Stimulation
}

\author{
Ursula Stäubli and Joey Scafidi \\ Center for Neural Science, New York University, New York, New York 10003
}

Previous studies in slices have shown that low-frequency stimulation at $5 \mathrm{~Hz}$, i.e., theta pulse stimulation (TPS), completely reverses long-term potentiation (LTP) in area CA1 when delivered within 1-2 min after induction but produces progressively less depotentiation at longer delays, until it has no longer any impact at 30 min after induction. The present study examined whether LTP in the freely moving rat exhibits a similar timedependent susceptibility to reversal. Adult male Long-Evans rats with bilateral stimulating electrodes activating collateral/ commissural projections to area CA1 were used. A $1 \mathrm{~min}$ episode of TPS, ineffective when applied to naive pathways, was found to permanently erase LTP when delivered to the test pathway either $30 \mathrm{sec}$ or $15 \mathrm{~min}$ after induction. Administered at a delay of $30 \mathrm{~min}$, however, the same treatment no longer had any impact on established LTP. Additional experiments examined the ability of shorter TPS episodes to erase LTP and found that a $30 \mathrm{sec}$ treatment was effective at $30 \mathrm{sec}$ but not $15 \mathrm{~min}$ after induction. When the duration of TPS was further reduced to $15 \mathrm{sec}$, a reversal was no longer obtained at any delay. These results provide the first demonstration that the limited vulnerability of LTP to reversal by TPS, originally observed in vitro, also holds true for LTP in the awake animal and occurs along the same time frame, supporting the notion that LTP stabilization mechanisms take less than 30 min to be complete.

Key words: depotentiation; in vivo; hippocampus; LTP reversal; consolidation; chronic recording; retrograde amnesia; memory; TPS; depression; forgetting
Behavioral studies have shown that various manipulations can disrupt the stable encoding of memory if applied shortly after initial learning and thereby produce retrograde amnesia (McGaugh, 1966; McGaugh et al., 1993). The question naturally arises whether long-term potentiation (LTP) passes through a similar consolidation period during which it is susceptible to disruption. The first evidence suggestive of a vulnerable phase for LTP came from a study showing that transient hypoxia occurring within a few minutes after high-frequency stimulation prevented the stable formation of LTP in hippocampal area CA1 but was ineffective at longer delays (Arai et al., 1990b). An alternative approach to address the reversibility of LTP is to examine activity-dependent depotentiation, defined as a lasting decrement in synaptic efficacy induced by activation of the same set of pathways that were tetanized previously. Most initial observations on depotentiation were done in vitro and used stimulation protocols that differed in frequency, duration, and time of reversal attempt across laboratories, making it difficult for a coherent picture to emerge (Fujii et al., 1991; Wexler and Stanton, 1993; Bashir and Collingridge, 1994; O’Dell and Kandel, 1994; Barr et al., 1995; Wagner and Alger, 1995). One study examined LTP reversal in area CA1 of freely moving rats and found that, of the two frequencies tested, $5 \mathrm{~Hz}$ was significantly more effective than $1 \mathrm{~Hz}$ (Stäubli and Lynch, 1990). That there might be an optimal frequency and time for reversing LTP was subsequently confirmed in hippocampal slices; a 30 sec episode of $5 \mathrm{~Hz}$ stimulation named theta pulse stimulation (TPS), delivered within $<1 \mathrm{~min}$ after LTP induction, completely reversed LTP, whereas $1 \mathrm{~Hz}$

\footnotetext{
Received March 30, 1999; revised July 16, 1999; accepted July 23, 1999.

This work was supported in part by the Whitehall Foundation (M97R05 to U.S.).

Correspondence should be addressed to Dr. Ursula Stäubli, Cortex Pharmaceuticals, Inc., 15231 Barranca Parkway, Irvine, CA 92618.

Copyright (C) 1999 Society for Neuroscience $\quad 0270-6474 / 99 / 198712-08 \$ 05.00 / 0$
}

stimulation had no impact and stimulation at $10 \mathrm{~Hz}$ was no more effective than TPS (Larson et al., 1993). That the reversal was optimally elicited by stimulation in the theta frequency range was attributed to the fact that it imitates endogenous hippocampal firing activity typically present during exploration (Ranck, 1973; Bland et al., 1980). Using TPS in follow-up experiments revealed that a $1 \mathrm{~min}$ episode $30 \mathrm{sec}$ after LTP induction produced a complete reversal but had increasingly less impact at longer delays and virtually no effect at $30 \mathrm{~min}$. Increasing the duration of TPS did not produce more depotentiation, whereas decreasing it reduced the degree of reversal (Stäubli and Chun, 1996a,b).

To assess the behavioral relevance of the above physiological findings, it is essential to determine whether the limited window of vulnerability to reversal observed in slices holds true for CA1 LTP in the awake animal. None of the recent studies on depotentiation in area CA1 has examined the time frame of reversibility or investigated whether the depression induced at a given time point is enduring and synapse-specific (Errington et al., 1995; Doyère et al., 1996; Doyle et al., 1997; Hölscher et al., 1997; Manahan-Vaughan, 1997). The present study focused on TPS and its ability to produce a homosynaptic reversal of LTP at increasing delays after induction. The goal was to establish whether the time course of reversal in the freely moving rat corresponds to that found in slices and to determine the minimum stimulation necessary to obtain the effect.

\section{MATERIALS AND METHODS}

Subjects. Twenty-six adult male Long-Evans rats, 3 months of age at the time of surgery, were used. The animals were housed individually and kept in a $12 \mathrm{hr}$ light/dark cycle with food and water available ad libitum. The animal care and use protocol for the present study was approved by the New York University Animal Welfare Committee.

Preparation for chronic hippocampal physiology. Preparation of animals with chronically implanted electrodes followed procedures essentially as described in a previous study (Stäubli and Scafidi, 1997). Subjects were 
deeply anesthetized with pentobarbital $(65 \mathrm{mg} / \mathrm{kg})$ and pretreated with atropine $(0.1 \mathrm{mg} / \mathrm{kg})$ to prevent excessive salivation. A Teflon-insulated platinum-iridium recording electrode $(75 \mu \mathrm{m})$ was lowered under stereotaxic guidance into stratum radiatum of hippocampal area CA1 (coordinates of $3.8 \mathrm{~mm}$ posterior and $2.5 \mathrm{~mm}$ lateral to bregma). Two Formvar-coated stainless steel monopolar stimulating electrode (125 $\mu \mathrm{m})$ were positioned into field CA3, one in each hemisphere (coordinates of $3.5 \mathrm{~mm}$ posterior and $3.0 \mathrm{~mm}$ lateral to bregma), to activate independent sets of Schaffer collateral and commissural projections converging on the population of dendrites sampled by the ipsilateral recording electrode. An indifferent electrode (125 $\mu \mathrm{m}$, Formvar-coated stainless steel) with $2 \mathrm{~mm}$ of insulation removed from the tip was lowered anterior to the hippocampus, and a stainless steel bone screw over the cerebellum served as a ground. The final depth of the electrodes was adjusted by maximizing the amplitude of the negative-going dendritic field EPSPs elicited by single stimulation pulses to the ipsilateral and contralateral stimulating electrode. After these steps, the leads of the electrodes were connected to a head stage that was permanently affixed to the rat's skull.

LTP induction. The rats were allowed $\sim 10 \mathrm{~d}$ for recovery before being acclimated to a chronic recording cage $(30 \times 30 \times 58 \mathrm{~cm})$ and the attachment of a recording lead to their head stage. Biphasic stimulation pulses were provided by a custom built computer-operated digital stimulator that allowed precise control of current intensity and pulse duration. Current intensity $(23-64 \mu \mathrm{A})$ and pulse width $(100-150 \mu \mathrm{sec})$ were adjusted to produce a response that was $50-60 \%$ of the maximum amplitude of the population spike-free response, which typically ranged between 7 and $8 \mathrm{mV}$. Recording signals were preamplified $10 \times$ via a field effect transistor operational amplifier built into the recording lead and fed into a second stage amplifier set to a gain of 10, with a bandpass of $1 \mathrm{~Hz}$ to $5 \mathrm{kHz}$. The evoked responses were monitored on a storage oscilloscope and fed into a personal computer running customized software with a Keithley Instruments (Cleveland, $\mathrm{OH}$ ) Metrabyte interface board, which digitized a $30 \mathrm{msec}$ sweep at $10 \mathrm{kHz}$. The initial slope and peak amplitude of each response was measured on-line, and the average waveform of groups of four successive responses was stored on disk for off-line analysis. Baseline evoked EPSPs were tested alternately on the two electrodes at $15-20 \mathrm{sec}$ intervals during baseline periods and after attempts to induce LTP-reverse LTP. Baseline periods were conducted daily during 30-45 min for at least $2 \mathrm{~d}$, and only those animals with $\leq 10 \%$ variability in size of the evoked response (initial slope and amplitude) over 2 consecutive days were used. Approximately $75 \%$ of the animals implanted met this criterion. LTP was induced on the subsequent day after $30 \mathrm{~min}$ of baseline recording. To elicit LTP, theta burst stimulation (TBS) consisting of two sets of five theta bursts separated by $30 \mathrm{sec}(2 \times 5 \mathrm{TBS})$, with each burst involving four pulses at $100 \mathrm{~Hz}$ and paced at $200 \mathrm{msec}$, was given to the test pathway. Current intensities and pulse widths were increased during TBS to elicit a very small $(<0.5 \mathrm{mV})$ population spike but otherwise were kept constant throughout the entire experiment.

LTP reversal. To determine whether the reversal of LTP is timedependent and, if so, whether the degree of depotentiation is contingent on the duration of the reversing stimulation, the following protocols were tested.

(1) A 1 min train of TPS, consisting of 300 single pulses paced at the period of the theta rhythm $(200 \mathrm{msec})$, was given to the test pathway 30 sec after TBS had been used to induce LTP in a group of five rats. Responses to both ipsilateral and contralateral pathway (test and control response) were monitored immediately thereafter for $\sim 2.5 \mathrm{hr}$ and again on subsequent days. If a reversal of LTP was obtained and still present at least $3 \mathrm{~d}$ later, then a second episode of TBS $(2 \times 5)$ was given to the same pathway, and the stability of the newly induced LTP was monitored for several days. This exact protocol was repeated in two additional groups of rats, except that the TBS-TPS interval was increased to 15 $(n=5)$ and $30(n=6) \mathrm{min}$, respectively. In all cases, the control pathway, which did not receive TBS and TPS, was monitored throughout the experiment to determine whether the reversal obtained in the test pathway was input-specific.

(2) The second set of experiments involved a new group of rats $(n=$ 5) and a TPS episode that was reduced in half to 150 pulses (30 sec episode). If a reversal of potentiation was obtained with TPS delivered 30 sec after LTP induction and still present $3 \mathrm{~d}$ later, then a second TBS treatment was given to the same pathway to reinstate LTP, followed 15 min later by attempts to reverse the potentiation with a second TPS episode of 150 pulses.

(3) The third series of experiments involved a new group of five rats but otherwise was identical to the set described immediately above, except that the TPS episode was reduced to 75 pulses (i.e., $15 \mathrm{sec}$ duration).

Statistics. All data are expressed as mean \pm SEM percentage baseline EPSP slope. Group means of averages for four consecutive responses were constructed, and effects were analyzed by conducting within-animal comparisons using the paired two-tailed $t$ test for average values collected over a specified 5-10 min period. Typically, the average response obtained during the last 10 min before TBS served as reference and was compared with the average response acquired during the last $5 \mathrm{~min}$ of the LTP recording period immediately preceding TPS (except for experiments involving a $30 \mathrm{sec}$ TBS-TPS interval). Similarly, the average response obtained during the last $10 \mathrm{~min}$ before TBS was compared with the average response collected during the first $5 \mathrm{~min}$ immediately after TPS, as well as with the very last $10 \mathrm{~min}$ of each recording period conducted on the same and subsequent days.

\section{RESULTS}

Consistent with earlier studies (Stäubli and Lynch, 1987, 1990; Stäubli and Scafidi, 1997), TBS reliably produced LTP in all the rats, and control responses remained unaffected by any of the high- and low-frequency stimulation treatment. Moreover, there was no evidence that prolonged low-frequency stimulation ever induced epileptiform activity or afterdischarges. (1) TPS of 300 pulses administered $30 \mathrm{sec}$ after LTP induction resulted in an immediate and complete reversal of potentiation that persisted for the duration of the $2.5 \mathrm{hr}$ test session and was still present 72 hr later. As illustrated in Figure $1 A$, the test response was significantly potentiated after TBS $\left(T_{(4)}=5.95 ; p<0.01\right)$ but was no longer different from baseline both immediately and $72 \mathrm{hr}$ after $\operatorname{TPS}\left(T_{(4)}=0.39 ; p>0.1\right.$; and $T_{(4)}=0.78 ; p>0.1$, respectively). No evidence of synaptic suppression of the control response $(n=$ 4 ) was found, as illustrated in Figure $1 B$ for a typical case (in one rat, the control response was not measurable). Three days after the reversal, TBS without subsequent TPS was administered to the same pathway, resulting in a significant potentiation that remained for $4 \mathrm{~d}$ (72 $\mathrm{hr}$ after TBS, $\left.T_{(4)}=5.36 ; p<0.01\right)$. In one rat, the response was lost on day 4 , and recording was therefore stopped.

Delaying the administration of TPS by 15 min again resulted in a rapid and complete LTP reversal, virtually identical to that observed with a $30 \mathrm{sec}$ TBS-TPS interval. As illustrated in Figure $2 A$, the test response in all five animals was significantly increased after TBS $\left(T_{(4)}=3.88 ; p<0.01\right)$ but was reset to baseline by TPS, an effect that took place immediately and persisted for several days $\left(T_{(4)}=0.89 ; p>0.1\right.$; and $T_{(4)}=1.5 ; p>0.1$, for immediate and $72 \mathrm{hr}$ post-TPS recording periods, respectively). On day 3 after TPS, a second TBS episode was administered to the test pathway, which resulted in significant potentiation that persisted for $4 \mathrm{~d}\left(2.5 \mathrm{hr}\right.$ after TBS, $T_{(4)}=6.13 ; p<0.01 ; 24 \mathrm{hr}$ after TBS, $T_{(4)}=9.86 ; p<0.01$; and $96 \mathrm{hr}$ after TBS, $\left.T_{(4)}=3.72 ; p<0.05\right)$. Control responses, which were present in four of the five rats in this group, again remained unaffected (see Fig. $2 B$ for a typical case).

When TPS was delivered $30 \mathrm{~min}$ after LTP, a reversal of potentiation was no longer obtained. Within-animal comparisons using the paired $t$ test for average values collected over the last 10 min before TPS with the last 10 min of that recording session was not statistically different $\left(T_{(5)}=1.25 ; p<0.1\right)$, indicating that TPS was not effective at depressing the potentiated synapses. As illustrated in Figure $3 A$, TBS administered to the test pathway induced a potentiation $\left(T_{(5)}=15.9 ; p<0.01\right)$ that persisted for 


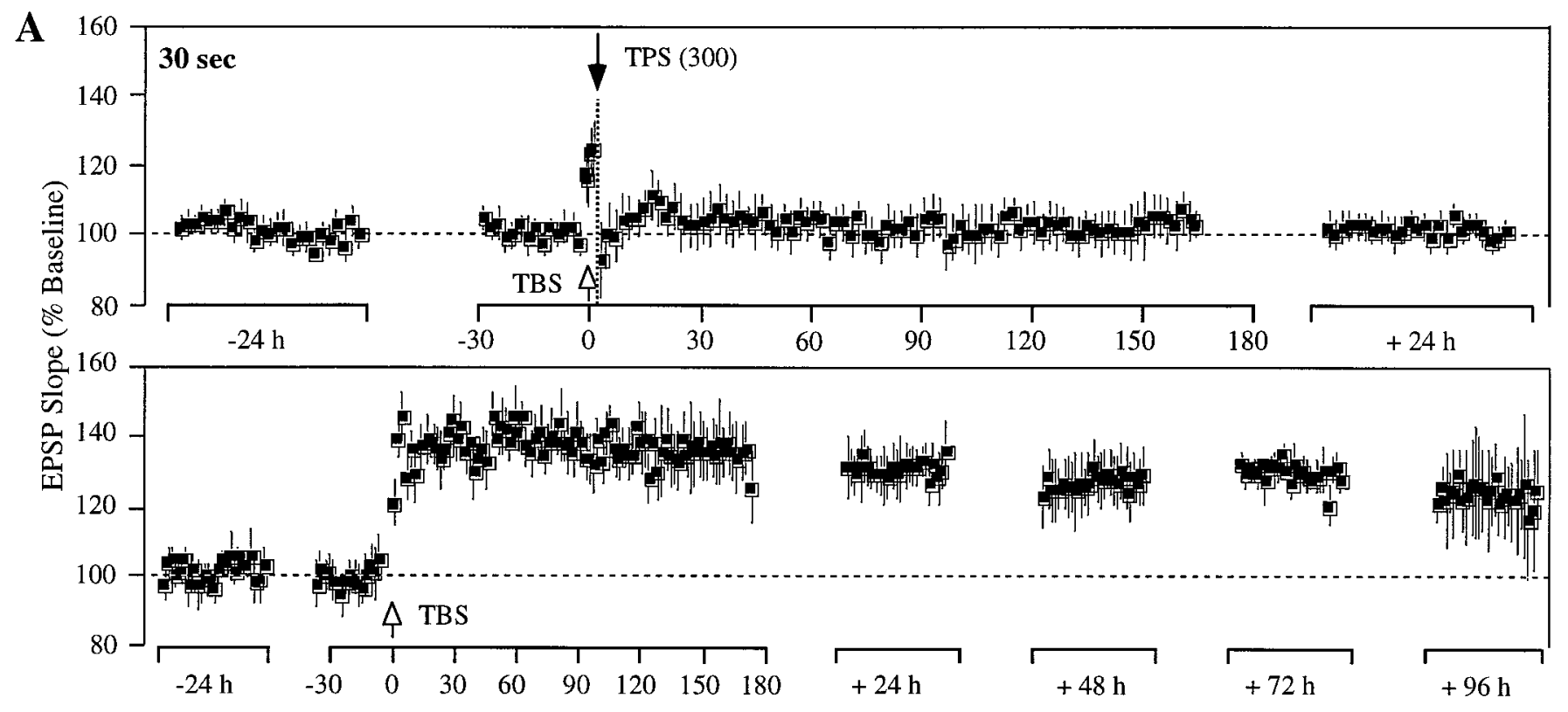

Time $(\mathrm{min} / \mathrm{hrs})$

B $\quad 30 \mathrm{sec}$

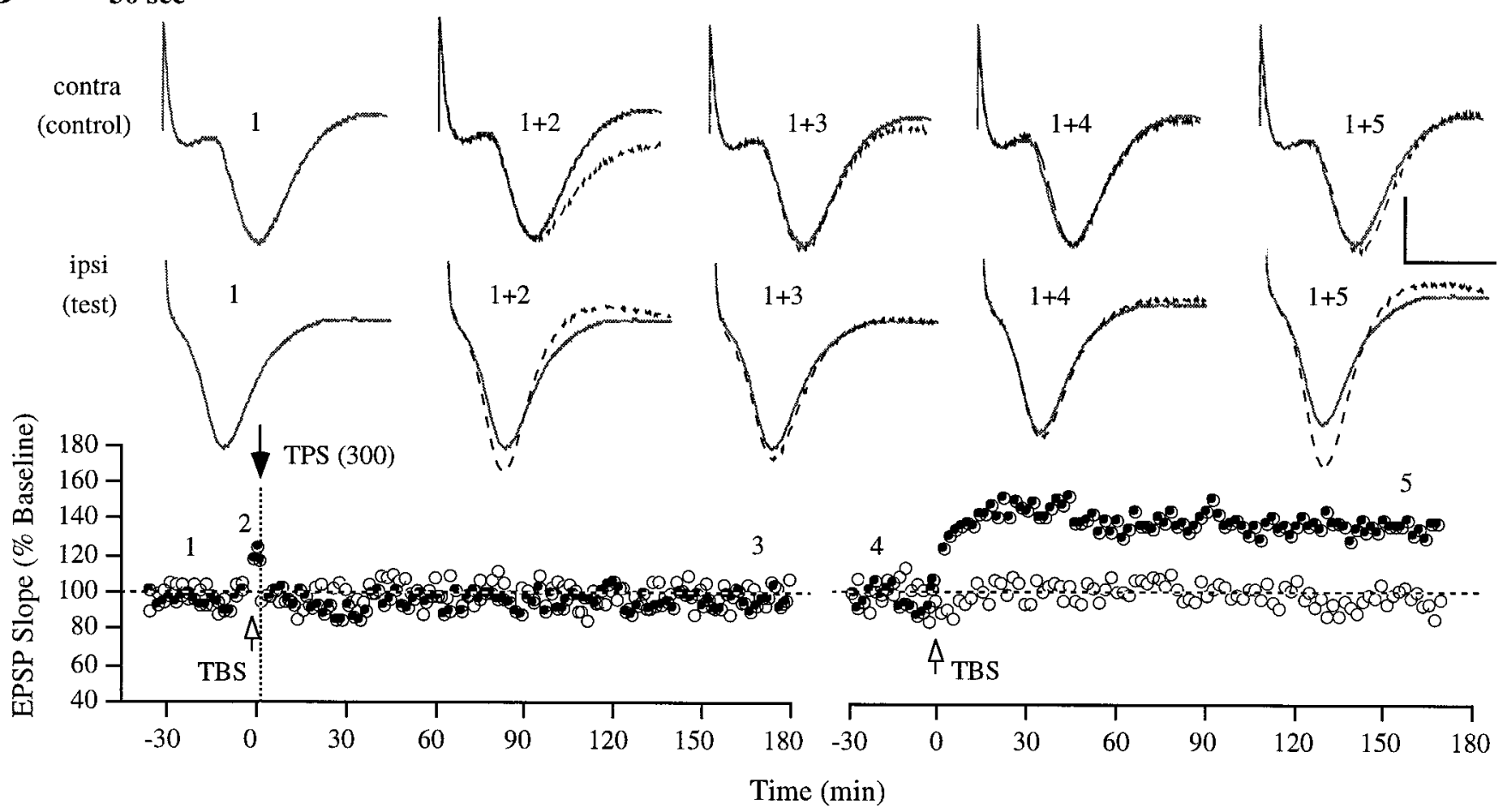

Figure 1. Stable reversal of LTP by theta pulse stimulation delivered $30 \mathrm{sec}$ after induction of LTP in area CA1 of the freely moving rat. $A$, Group data from five animals showing that a $1 \mathrm{~min}$ train of TPS administered $30 \mathrm{sec}$ after TBS caused a lasting reversal of LTP. A second TBS episode administered $3 \mathrm{~d}$ after TPS produced a potentiation effect that was stable for the $4 \mathrm{~d}$ of recording thereafter. Each data point represents the group mean \pm SEM (averages of 4 successive responses per animal and data point, except for the 4 individual responses within the TBS-TPS interval) of the initial slope of the dendritic field potential expressed as percentage of the baseline. $B$, Individual representative experiment on treatment days from one of the five rats shown in $A$. Top, Representative population EPSPs of control and test pathway measured at different time points during the experiment, as indicated by the numbers in the graph below. The solid waveforms indicate the size of the baseline EPSPs, and the superimposed dotted waveforms represent control and test responses recorded at the specified times. Each waveform represents the average of four successive responses to single-pulse stimulation at 0.05 Hz. Calibration: $3 \mathrm{mV}, 10 \mathrm{msec}$. 


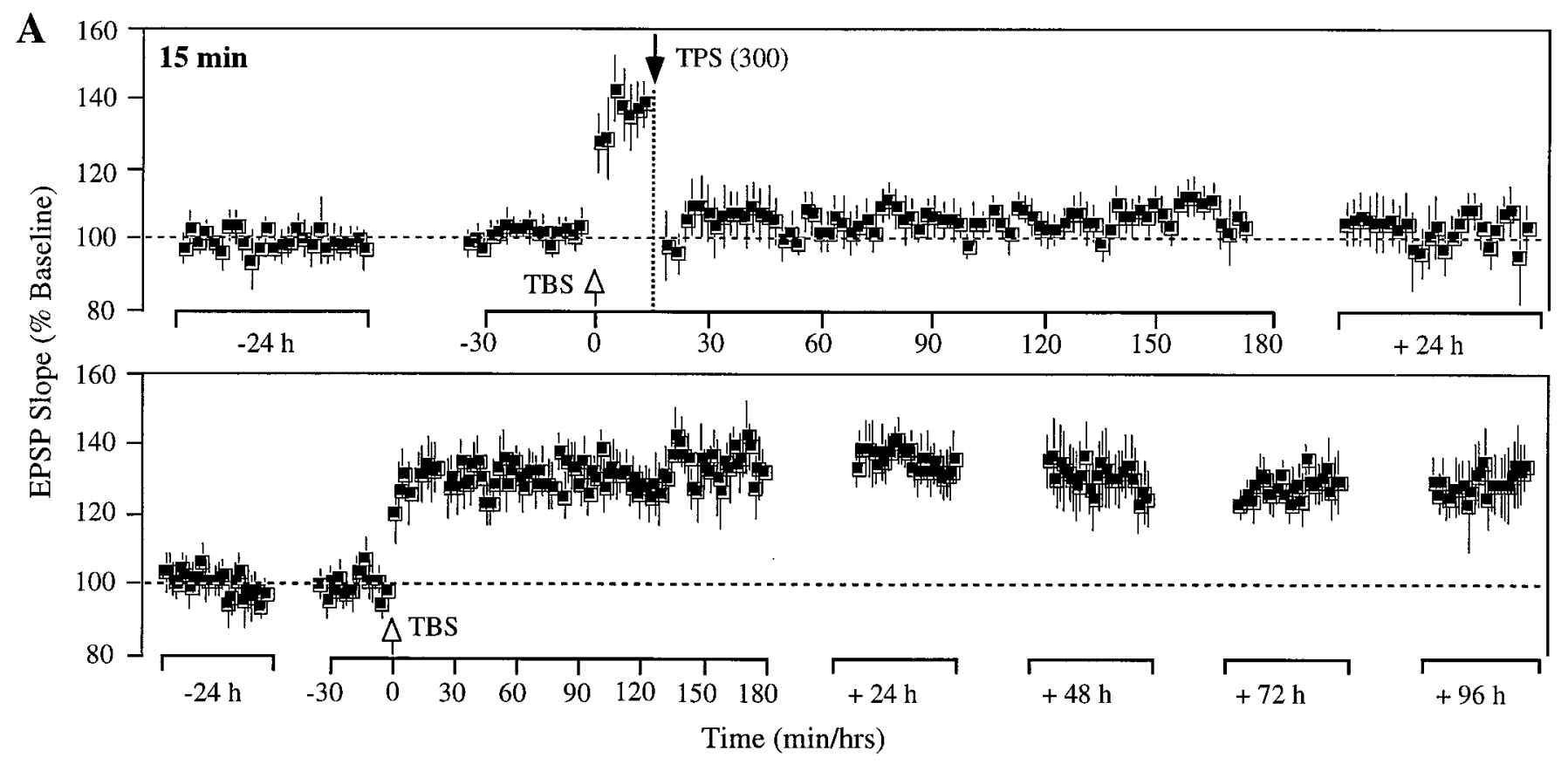

B

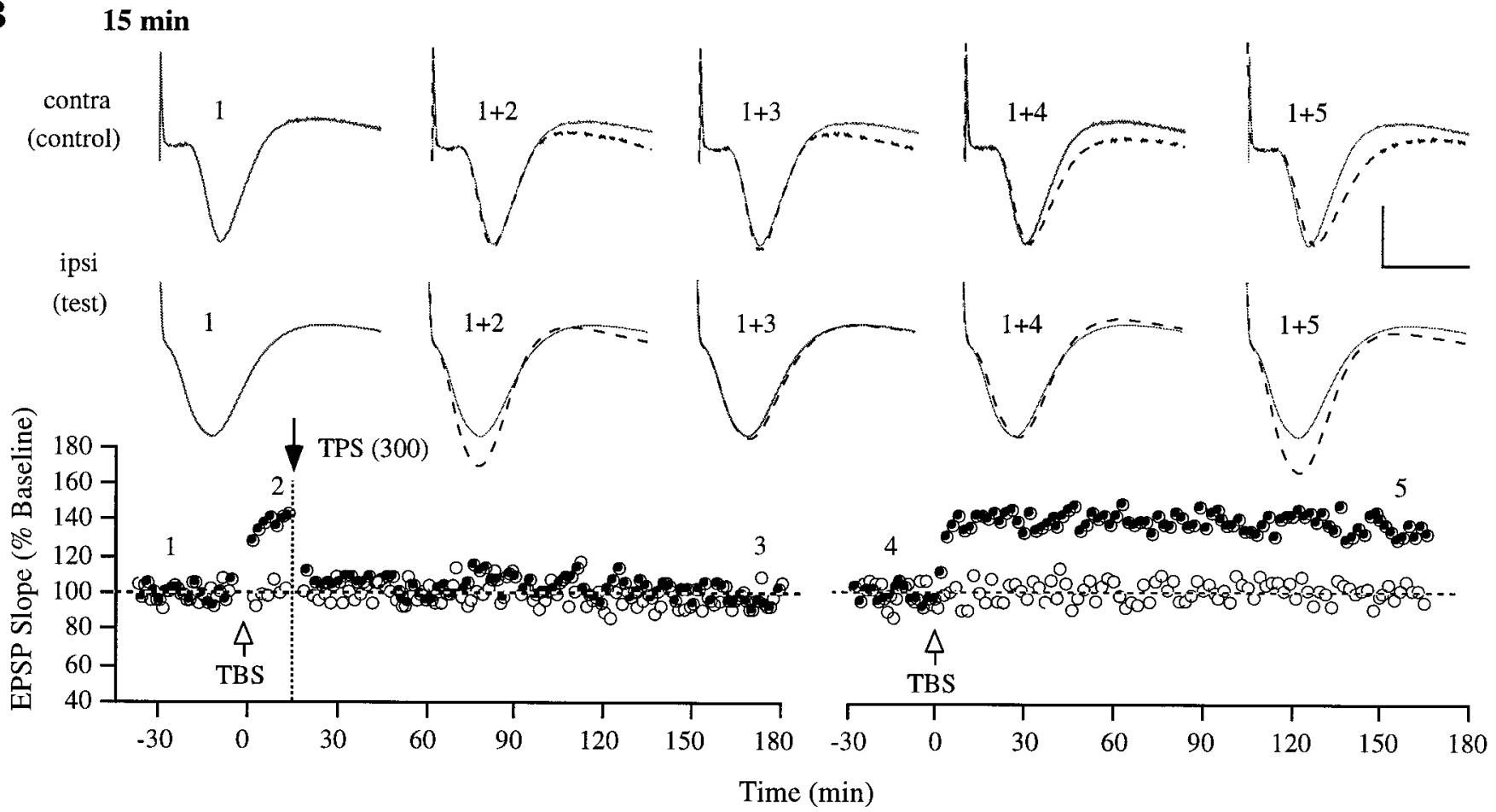

Figure 2. Stable reversal of LTP by theta pulse stimulation delivered $15 \mathrm{~min}$ after LTP induction in area CA1 of the freely moving rat. $A$, Group data from five animals showing that a 1 min train of TPS administered 15 min after TBS caused a lasting reversal of LTP. A second TBS episode administered $3 \mathrm{~d}$ after TPS produced a potentiation effect that was stable for the $4 \mathrm{~d}$ of recording thereafter. Each data point represents the group mean \pm SEM (averages of 4 successive responses per animal and data point) of the initial slope of the dendritic field potential expressed as percentage of the baseline. $B$, Individual representative experiment on treatment days from one of the five rats shown in $A$. Top, Representative population EPSPs of control and test pathway measured at different time points during the experiment, as indicated by the numbers in the graph below. The solid waveforms indicate the size of the baseline EPSPs, and the superimposed dotted waveforms represent control and test responses recorded at the specified times. Each waveform represents the average of four successive responses to single-pulse stimulation at $0.05 \mathrm{~Hz}$. Calibration: $3 \mathrm{mV}, 10 \mathrm{msec}$. 
A

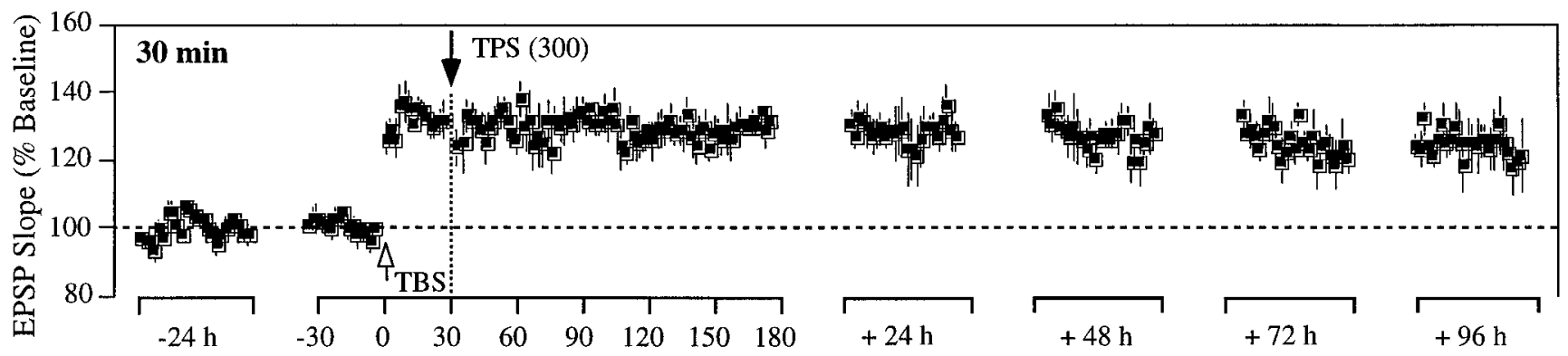

Time $(\mathrm{min} / \mathrm{hrs})$

B

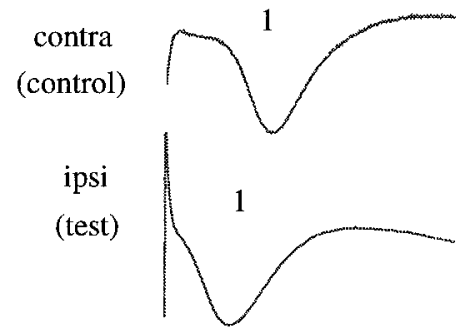

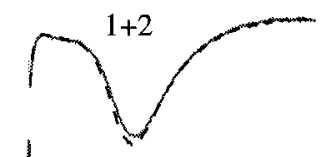

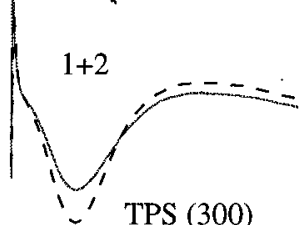

TPS (300)
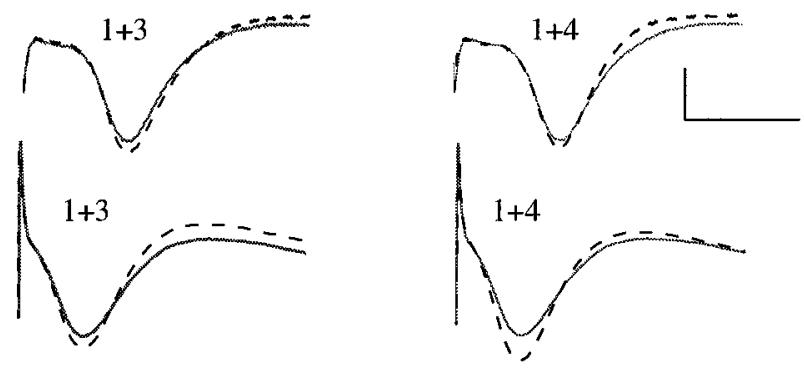

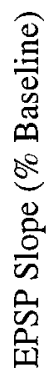
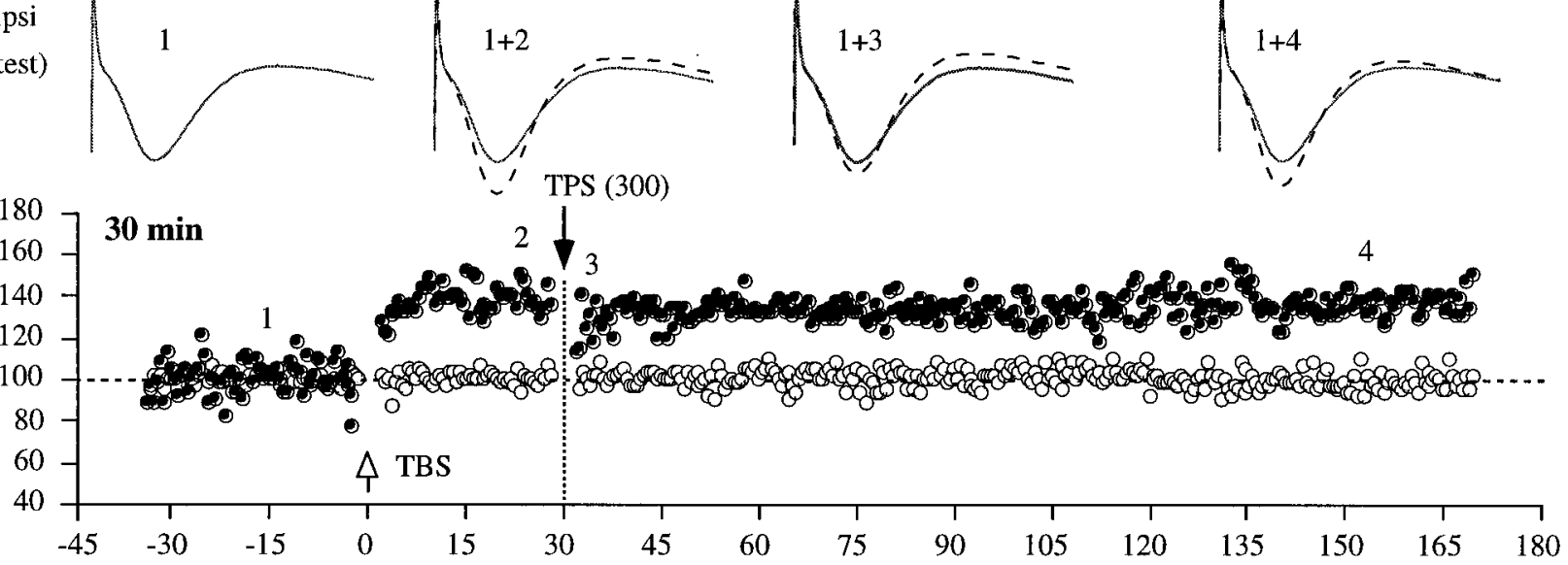

$2 \int_{0} 3$

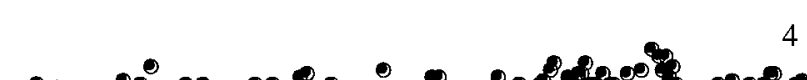

4

Time $(\min )$

Figure 3. Failure to obtain LTP reversal when theta pulse stimulation is administered 30 min after induction. $A$, Group data from six animals illustrating that LTP was not affected by a 1 min train of TPS delivered 30 min after induction and continued to persist for the $4 \mathrm{~d}$ of recording thereafter. Each data point represents the group mean \pm SEM (averages of 4 successive responses per pathway and data point) of the initial slope of the dendritic field potential expressed as percentage of the baseline. $B$. Individual representative experiment from one of the six rats shown in $A$. Top, Representative population EPSPs in control and test pathway measured at different time points during the experiment, as indicated by the numbers in the graph below. The solid waveforms indicate the size of the baseline EPSPs, and the superimposed dotted waveforms represent control and test responses recorded at the specified times. Each waveform represents the average of four successive responses to single-pulse stimulation at $0.05 \mathrm{~Hz}$. Calibration: $3 \mathrm{mV}, 10 \mathrm{msec}$.

the $4 \mathrm{~d}$ of recording $\left(T_{(5)}=7.82 ; p<0.01\right)$. In two of the rats, TPS caused a temporary depression of the potentiated response to levels above baseline, which recovered to the previously potentiated level within $5 \mathrm{~min}$ in both animals (see Fig. $3 B$ for an example). Control responses were present in three of the six rats and again remained unaffected by TPS given to the test pathway.

(2) Reducing the duration of TPS from $1 \mathrm{~min}$ to $30 \mathrm{sec}$ (150 as opposed to 300 pulses) resulted in a complete reversal of potentiation when delivered $30 \mathrm{sec}$ after TBS. As shown in Figure 4, the test response was significantly potentiated by TBS $\left(T_{(4)}=\right.$ $4.52 ; p<0.01$ ) but decayed back to baseline after TPS. In three of the rats, the return to baseline occurred gradually over the course of 15-30 min, whereas in the remaining two subjects, the reversal was complete at once. Although the average response was above baseline in the first 5 min after TPS, the difference was not significant $\left(T_{(4)}=2.1 ; p>0.05\right)$. At the end of the $2.5 \mathrm{hr}$ recording session, the test response was indistinguishable from baseline $\left(T_{(4)}=0.23 ; p>0.1\right)$. On post-TPS day 3 , a second episode of TBS was delivered to the same pathway, causing a significant increase in the response $\left(T_{(4)}=9.24 ; p<0.01\right)$. LTP was monitored for $15 \mathrm{~min}$, after which a $30 \mathrm{sec}$ treatment of TPS was administered. However, unlike previously, no reversal of potentiation was obtained (10 min before TPS vs last $10 \mathrm{~min}$ of treatment day; $\left.T_{(4)}=0.93 ; p>0.1\right)$, indicating that a $15 \mathrm{~min}$ TBS-TPS interval exceeds the time frame during which LTP is susceptible to reversal by a reduced TPS treatment. The test response remained potentiated for the $4 \mathrm{~d}$ of recording afterward. Four of the five rats in this group exhibited a response in the control pathway, which remained unaffected by both TPS treatments.

(3) When the duration of TPS was further reduced to $15 \mathrm{sec}$ (75 pulses), it became ineffective at reversing LTP, even when administered at the shortest TBS-TPS interval, i.e., at $30 \mathrm{sec}$ after LTP induction. As illustrated in Figure 5, the test response was signif- 


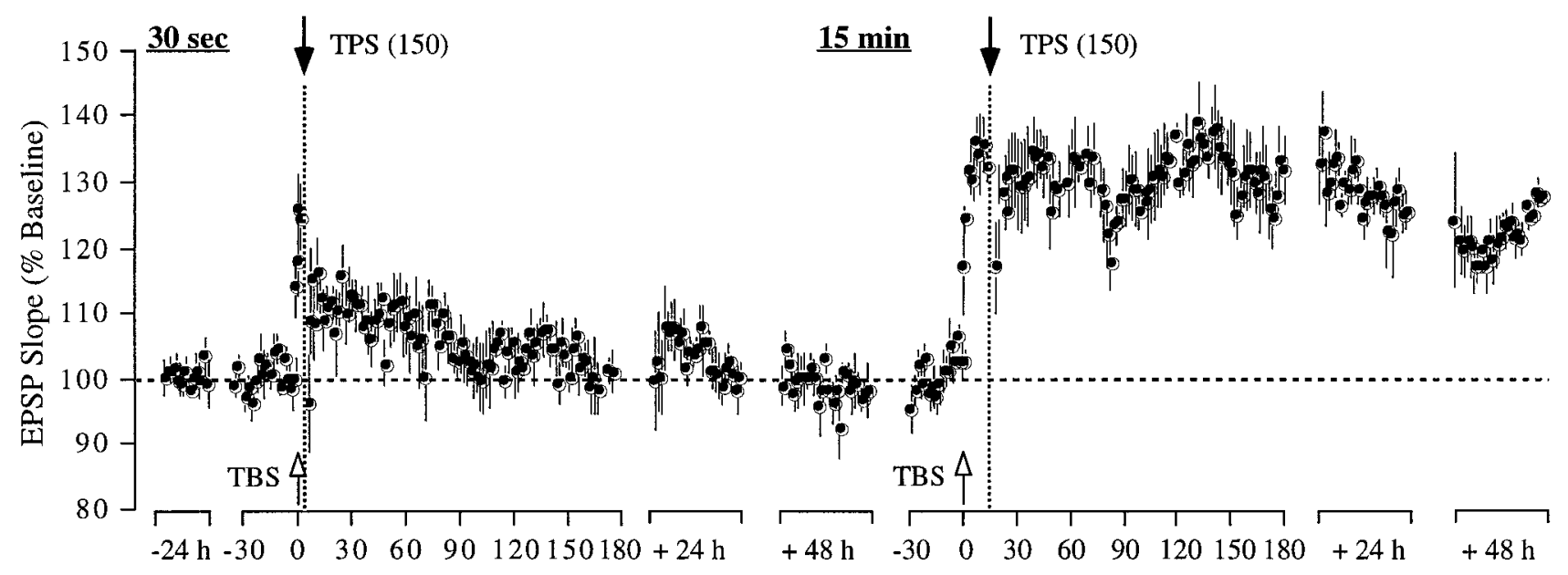

Time $(\mathrm{min} / \mathrm{hrs})$

Figure 4. Reducing the duration of theta pulse stimulation shortens the time frame during which the stimulation is effective at reversing LTP. Group data from five animals illustrating that a $30 \mathrm{sec}$ train of TPS (150 pulses) was sufficient to cause a persistent reversal of LTP when administered 30 sec after induction but failed to have any impact on LTP when administered 15 min after LTP induction. Each data point represents the group mean \pm SEM (averages of 4 successive responses per animal and data point, except for the 4 individual responses within the $30 \mathrm{sec}$ TBS-TPS interval) of the initial slope of the dendritic field potential expressed as percentage of the baseline.

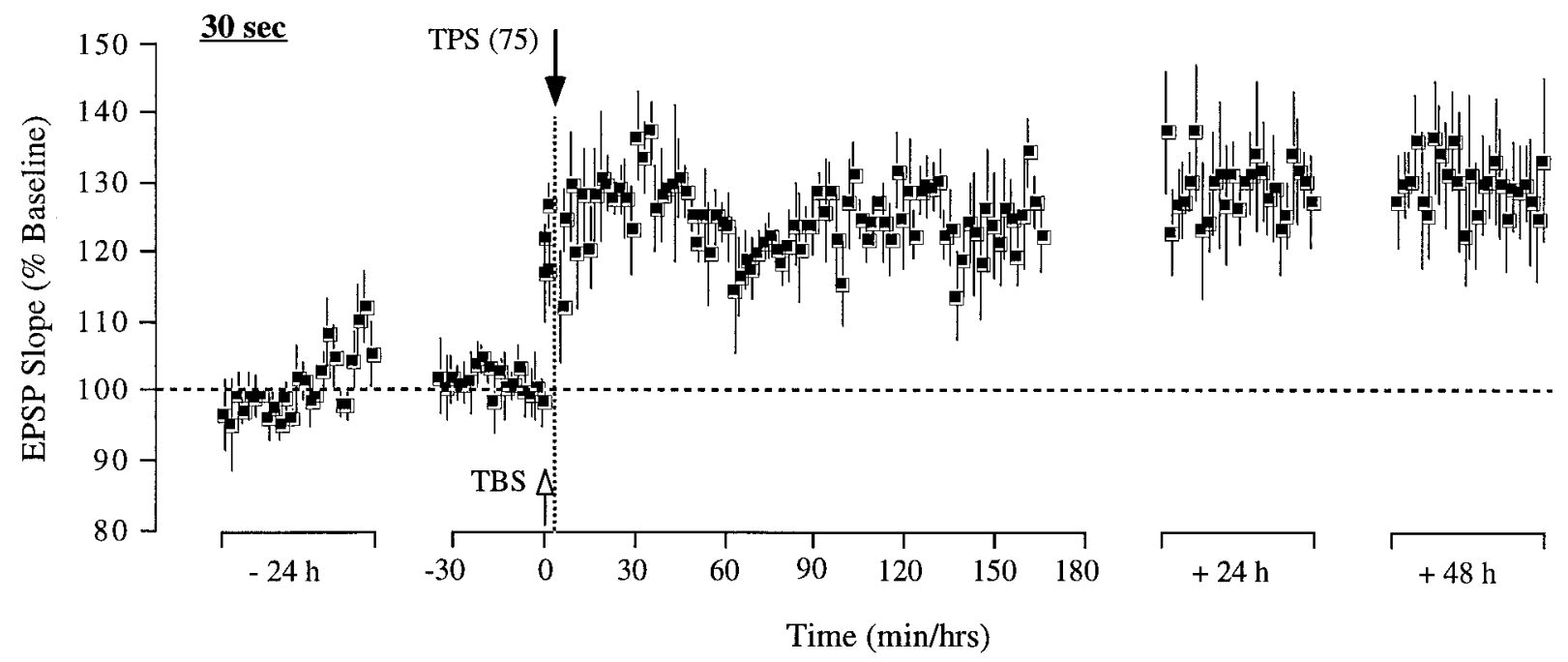

Figure 5. Fifteen seconds of theta pulse stimulation is too short to produce a reversal of LTP. Group data from five animals illustrating that a reduction in TPS duration to $15 \mathrm{sec}$ (75 pulses) eliminates its capacity to reverse LTP. Each data point represents the group mean \pm SEM (averages of 4 successive responses per animal and data point, except for the 4 individual responses within the $30 \mathrm{sec}$ TBS-TPS interval) of the initial slope of the dendritic field potential expressed as percentage of the baseline.

icantly enhanced by TBS in the first $30 \sec \left(T_{(4)}=5.38 ; p<0.01\right)$ and, despite the delivery of TPS, remained potentiated for the next $48 \mathrm{hr}\left(T_{(4)}=7.7 ; p<0.01\right)$. Control responses, which were present in three of the five rats, remained unaffected by TPS to the test pathway.

Further analysis using a one-way ANOVA with repeated measures of the normalized EPSP slope values revealed that, for the groups of rats that exhibited TPS-induced LTP reversal, there was no significant difference in the amount of reversal obtained during the last $10 \mathrm{~min}$ of recording on treatment day $\left(F_{(2,12)}=\right.$ $0.82 ; p>0.1)$. Also, the same statistical comparisons were conducted to determine whether the amount of potentiation of the EPSP slope during the last $10 \mathrm{~min}$ on treatment day differed among the groups of rats that either exhibited no reversal or when no TPS was administered to the potentiated synapses $\left(F_{(4,21)}=\right.$ $0.79 ; p>0.1)$.

\section{DISCUSSION}

The present findings show that LTP in area CA1 of the freely moving rat exhibits a time window of vulnerability of $<30 \mathrm{~min}$ after induction, after which it becomes resistant to disruption by TPS. The same 1 min stimulation episode had no effect when administered before LTP induction, did not cause generalized disturbances of hippocampal activity, and equally important, did not affect heterosynaptic, control-evoked potentials. LTP reversal always occurred rapidly and persisted for several days. The lack of any depressive action of TPS on pre-LTP responses, although 
some percentage of the synapses were presumably already potentiated by earlier learning experiences, is consistent with our finding that TPS-induced reversal only occurs for a relatively brief interval after induction of LTP.

The duration of the vulnerable phase observed in the present study is virtually identical to that established earlier in hippocampal slices using the same TPS protocol (Stäubli and Chun, 1996a). However, the in vivo results differ with respect to the magnitude of the effect. In the intact hippocampus, LTP reversal was typically expressed in an all-or-none manner, i.e., the potentiation always fully reversed to baseline value when TPS was delivered within the vulnerable period. In slices, on the other hand, the degree of depotentiation progressively declined as the interval between LTP induction and reversal attempt approached $30 \mathrm{~min}$.

It has been reported recently that LTP in the dentate gyrus of anesthetized rats exhibits a vulnerable phase of $<10$ min during which it can be reversed by low-frequency stimulation at $5 \mathrm{~Hz}$ but not $1 \mathrm{~Hz}$ (Martin, 1998). Thus, the factors regulating the reversibility of LTP in different hippocampal subfields may vary with regard to time dependence and magnitude of effect, depending on cell type and/or state of consciousness of the animal. Related to the above may also be the finding that 10 stimulation bursts delivered sequentially on the negative phase of the sensoryevoked theta rhythm in anesthetized rats partially reverse CA1 LTP established $30 \mathrm{~min}$ earlier (Hölscher et al., 1997). That naturally occurring hippocampal activity in the theta frequency range may indeed contribute to experience-dependent erasure of LTP is suggested in a recent report, showing that exploration of a novel environment causes a reversal of LTP within a defined time window of $1 \mathrm{hr}$. Exploration of the novel environment was accompanied by increased electroencephalic activity in the theta range and was selectively associated with the processing of new information (Xu et al., 1998). The existence of an experiencedependent LTP reversal effect within a defined time window raises the possibility that loss of recent memory can result from an active process triggered by physiological patterns associated with particular behavioral states.

Chronic unit recording studies in our and other laboratories have established that hippocampal pyramidal cells in behaving rats fire in single-spike mode synchronized to ongoing theta activity for periods lasting several seconds (Bland et al., 1980; S. Wiebe and U. Stäubli, unpublished observations). The present study revealed that the minimum TPS necessary to trigger a reversal consists of 150 pulses, a treatment only effective if applied at $30 \mathrm{sec}$ but not $15 \mathrm{~min}$ after induction. A $15 \mathrm{sec}$ TPS episode, on the other hand, was too short to cause depotentiation at any delay. This is not to say that $15 \mathrm{sec}$ of TPS would not have been sufficient to reverse LTP of lesser magnitude, i.e., the kind of submaximal potentiation obtained when using fewer than 10 theta bursts (Larson and Lynch, 1986). No attempts were made to apply TPS for $>60 \mathrm{sec}$, because the amount of reversal obtained by a 3 min train in slices was found indistinguishable from that obtained with a 1 min train (Stäubli and Chun, 1996a). Moreover, a main objective of the present study was to determine the minimum TPS duration necessary to reverse LTP. If electrical stimulation-induced LTP represents the type of increase in synaptic strength that naturally occurs to a much smaller degree at a given synapse during learning, then it is perhaps not unreasonable to assume that a few seconds worth of endogenous theta pulse firing activity might be sufficient to reverse recent learninginduced increments in synaptic strength.

The observation that TPS becomes increasingly less effective as the time since induction progresses until LTP is no longer reversible at 30 min suggests that the low-frequency stimulation triggers a mechanism that directly antagonizes the effect of consolidation chemistries and is effective up to the completion of LTP stabilization. An indication as to the type of chemistries that might be involved in stabilization comes from in vitro studies showing that peptides that block ligand binding by integrin adhesion receptors prevent the stabilization of LTP, without affecting its initial development or baseline physiology (Stäubli et al., 1990; Xiao et al., 1991), and, most importantly, cause a steady decay of LTP, even when applied after induction (Bahr et al., 1997). Moreover, integrin antagonism was found to produce progressively less depotentiation as the interval between LTP induction and drug application increased, until it had no longer any impact at $30 \mathrm{~min}$ (Stäubli et al., 1998). This close similarity between the in vitro time courses for LTP reversal by integrin antagonism versus TPS implies that integrin activation may be a critical step in the consolidation of LTP and suggests that the two effects may be linked. What remains to be tested is whether intracerebral injections of antagonist into freely moving animals affects recently induced potentiation over the same 15-30 min time frame as TPS.

A possible route whereby TPS could deactivate integrins and thereby disrupt the stabilization of recently induced LTP is provided by adenosine receptors. Specifically, prolonged lowfrequency pulse stimulation at $5 \mathrm{~Hz}$, but not brief high-frequency burst stimulation, was found to preferentially cause the release of adenosine at synaptic sites (Cunha et al., 1996). Moreover, adenosine receptors have been implicated in suppressing integrin activation (Thiel et al., 1996). Linked to the above is evidence that application of adenosine within a limited time after induction reverses LTP (Arai et al., 1990a), and blockade of adenosine A1 receptors prevents depotentiation by TPS (Larson et al., 1993; Stäubli and Chun, 1996b). Combined, the above collection of results support the hypothesis that TPS, via its stimulatory action on adenosine release, suppresses the mobilization of integrins and thereby prevents the stabilization of changes in synaptic anatomy responsible for the persistence of LTP. It remains for future experiments to test the strength of the above conjecture.

In a more general sense, the existence of a time-dependent LTP reversal effect in the intact and awake hippocampus raises issues regarding its possible role in memory. Establishment of a relationship between the two would require comparisons of how the reversal of LTP in freely moving rats affects recently encoded memories. However, the difficulty with such an experiment is to establish that a sufficient number of synapses recruited by the LTP stimulating electrode are the same as those mediating the learning task. Perhaps more pertinent to the above question is the observation that the post-trial consolidation period during which newly acquired memories are susceptible to disruption by temporary inactivation of hippocampal processes (excluding lesions) is typically on the order of $15 \mathrm{~min}$ to $<1 \mathrm{hr}$ (Duncan, 1949; Riccio et al., 1968; Popik et al., 1994). That two very different phenomena, such as LTP and memory, exhibit vulnerable phases of very similar duration is intriguing and encourages the suggestion that the lasting encoding of memory and the stabilization of LTP are both regulated by the same cellular chemistries.

\section{REFERENCES}

Arai A, Kessler M, Lynch G (1990a) The effects of adenosine on the development of long-term potentiation. Neurosci Lett 119:41-44.

Arai A, Larson J, Lynch G (1990b) Anoxia reveals a vulnerable period in the development of long-term potentiation. Brain Res 511:353-357.

Bahr B, Stäubli U, Xiao P, Chun D, Xi Z-X, Esteban ET, Lynch G (1997) 
Arg-Gly-Asp-Ser selective adhesion and the stabilization of LTP: pharmacological studies and the characterization of a candidate matrix receptor. J Neurosci 17:1320-1329.

Barr DS, Lambert NA, Hoyt KL, Moore SD, Wilson WA (1995) Induction and reversal of long-term potentiation by low- and high-intensity pattern stimulation. J Neurosci 15:5402-5410.

Bashir ZI, Collingridge GL (1994) An investigation of depotentiation of long-term potentiation in the CA1 region of the hippocampus. Exp Brain Res 100:437-443.

Bland BH, Andersen P, Ganes T, Sveen O (1980) Automated analysis of rhythmicity of physiologically identified hippocampal formation neurons. Exp Brain Res 38:205-219.

Cunha RA, Vizi ES, Ribeiro JA, Sebastiao AM (1996) Preferential release of ATP and its extracellular catabolism as a source of adenosine upon high- but not low-frequency stimulation of rat hippocampal slices. J Neurochem 67:2180-2187.

Doyère V, Errington ML, Laroche S, Bliss TVP (1996) Low-frequency trains of paired stimuli induce long-term depression in area CA1 but not in dentate gyrus of the intact rat. Hippocampus 6:52-75.

Doyle CA, Cullen WK, Rowan MJ, Anwyl R (1997) Low-frequency stimulation induces homosynaptic depotentiation but not long-term depression of synaptic transmission in the adult anaesthetized and awake rat hippocampus in vivo. Neuroscience 77:75-85.

Duncan CP (1949) The retroactive effect of electroshock on learning. J Comp Physiol Psychol 42:32-44.

Errington ML, Bliss TVP, Richter-Levin G, Yenk K, Doyère V, Laroche S (1995) Stimulation at $1-5 \mathrm{~Hz}$ does not produce long-term depression or depotentiation in the hippocampus of the adult rat in vivo. J Neurophysiol 74:1793-1799.

Fujii S, Saito K, Miyakawa H, Ito K, Kato H (1991) Reversal of longterm potentiation (depotentiation) induced by tetanus stimulation of the input to CA1 neurons of guinea pig hippocampal slices. Brain Res 555:112-122.

Hölscher C, Anwyl R, Rowan MJ (1997) Stimulation on the positive phase of hippocampal theta rhythm induces long-term potentiation that can be depotentiated by stimulation on the negative phase in area CA1 in vivo. J Neurosci 17:6470-6477.

Larson J, Lynch G (1986) Induction of synaptic potentiation in hippocampus by patterned stimulation involves two events. Science 232:985-988.

Larson J, Xiao P, Lynch G (1993) Reversal of LTP by theta frequency stimulation. Brain Res 600:97-102.

Manahan-Vaughan D (1997) Group 1 and 2 metabotropic glutamate receptors play differential roles in hippocampal long-term depression and long-term potentiation in freely moving rats. J Neurosci 17:3303-3311.

Martin SJ (1998) Time-dependent reversal of dentate LTP by $5 \mathrm{~Hz}$ stimulation. NeuroReport 19:3775-3781.
McGaugh JL (1966) Time-dependent processes in memory storage. Science 153:1351-1358.

McGaugh JL, Introini-Collison IB, Cahill LF, Castellano C, Dalmaz C, Parent MB, Williams CL (1993) Neuromodulatory systems and memory storage: role of the amygdala. Behav Brain Res 58:81-90.

O'Dell TJ, Kandel ER (1994) Low-frequency stimulation erases LTP through an NMDA receptor-mediated activation of protein phosphatases. Learn Mem 1:129-139.

Popik P, Mamczarz J, Vetulani J (1994) The effect of electroconvulsive shock and nifedipine on spatial learning and memory in rats. Biol Psychiatry 35:864-869.

Ranck Jr JB (1973) Studies on single neurons in dorsal hippocampal formation and septum in unrestrained rats. I. Behavioral correlates and firing repertoires. Exp Neurol 41:461-531.

Riccio DC, Hodges LA, Randall PK (1968) Retrograde amnesia produced by hypothermia in rats. J Comp Physiol Psychol 66:618-622.

Stäubli U, Chun D (1996a) Factors regulating the reversibility of longterm potentiation. J Neurosci 16:853-860.

Stäubli U, Chun D (1996b) Proactive and retrograde effects on LTP produced by theta pulse stimulation: mechanisms and characteristics of LTP reversal in vitro. Learn Mem 3:96-105.

Stäubli U, Lynch G (1987) Stable hippocampal long-term potentiation elicited by "theta" pattern stimulation. Brain Res 435:227-234.

Stäubli U, Lynch G (1990) Stable depression of potentiated synaptic responses in the hippocampus with $1-5 \mathrm{~Hz}$ stimulation. Brain Res 513:113-118

Stäubli U, Scafidi J (1997) Studies on long-term depression in area CA1 of the anesthetized and freely moving rat. J Neurosci 17:4820-4828.

Stäubli U, Vanderklish P, Lynch G (1990) An inhibitor of integrin receptors blocks long-term potentiation. Behav Neural Biol 53:1-5.

Stäubli U, Chun D, Lynch G (1998) Time-dependent reversal of longterm potentiation by an integrin antagonist. J Neurosci 18:3460-3469.

Thiel M, Chambers JD, Chouker A, Fischer S, Zourelidis C, Bardenheuer HJ, Arfors KE, Peter K (1996) Effect of adenosine on the expression of beta(2) integrins and L-selectin of human polymorphonuclear leukocytes in vitro. J Leukoc Biol 59:671-682.

Wagner JJ, Alger BE (1995) GABAergic and developmental influences on homosynaptic LTD and depotentiation in rat hippocampus. J Neurosci 15:1577-1586.

Wexler EM, Stanton PK (1993) Priming of homosynaptic long-term depression in hippocampus by previous synaptic activity. NeuroReport 4:591-594.

Xiao P, Bahr BA, Stäubli U, Vanderklish PW, Lynch G (1991) Evidence that matrix recognition contributes to stabilization but not induction of LTP. NeuroReport 2:461-464.

Xu L, Anwyl R, Rowan MJ (1998) Spatial exploration induces a persistent reversal of long-term potentiation in rat hippocampus. Nature 394:891-894. 